\title{
Preliminary design of CERN Future Circular Collider tunnel: first evaluation of the radiation environment in critical areas for electronics
}

\author{
Angelo Infantino*, Rubén García Alía, Maria Ilaria Besana, Markus Brugger and Francesco Cerutti \\ European Organization for Nuclear Research (CERN), Geneva 23, Geneva CH-1211, Switzerland
}

\begin{abstract}
As part of its post-LHC high energy physics program, CERN is conducting a study for a new proton-proton collider, called Future Circular Collider (FCC-hh), running at center-of-mass energies of up to $100 \mathrm{TeV}$ in a new $100 \mathrm{~km}$ tunnel. The study includes a 90-350 GeV lepton collider (FCC-ee) as well as a lepton-hadron option (FCC-he). In this work, FLUKA Monte Carlo simulation was extensively used to perform a first evaluation of the radiation environment in critical areas for electronics in the FCC-hh tunnel. The model of the tunnel was created based on the original civil engineering studies already performed and further integrated in the existing FLUKA models of the beam line. The radiation levels in critical areas, such as the racks for electronics and cables, power converters, service areas, local tunnel extensions was evaluated.
\end{abstract}

\section{Introduction}

\subsection{From the LHC era to FCC}

The main goal of CERN's mission is pushing back the frontiers of human knowledge and, in doing so, drive innovation, develop forefront technologies, stimulate international collaboration and inspire tomorrow's leaders in science and technology. This mission is reached particularly through fundamental research in physics. In the last years, the Large Hadron Collider (LHC) allowed to push back the frontiers of human knowledge as anyone before bringing to the discovery of the Higgs boson in 2012, from the ATLAS and CMS experiments. The LHC and its high-luminosity upgrade, the HL-LHC, have an exciting physics program which extends through the mid 2030's [1].

The Future Circular Collider (FCC) is an integral conceptual design study for post-LHC particle accelerator options in a global context. The study is conducted within a collaboration that is open to scientific institutes and companies of any size from all nations and it currently counts 70 institutions spread in 26 countries. The FCC study has an emphasis on proton-proton collider (FCChh) but includes also a 90-350 GeV lepton collider (FCCee), seen as a potential intermediate step, as well as a lepton-hadron option (FCC-he) [2]. The FCC study is exploring the potential of hadron and lepton circular colliders, performing an in-depth analysis of infrastructure and operation concepts and considering the technology research and development programs that would be required to build a future circular collider. The need for building such large circular hadron collider is to reach energy levels far beyond the range of the LHC to provide access to the direct production of new particles with masses up to tens of $\mathrm{TeV}$, as well as to obtain much increased rates for phenomena in the sub-TeV mass range, with the corresponding greatly improved precision [1].

The goal of FCC-hh is to provide proton-proton collisions at a centre-of-mass energy of $100 \mathrm{TeV}$. Assuming a nominal dipole field of $16 \mathrm{~T}$, such a machine would have a circumference of the order of $100 \mathrm{~km}$ (Figure 1).

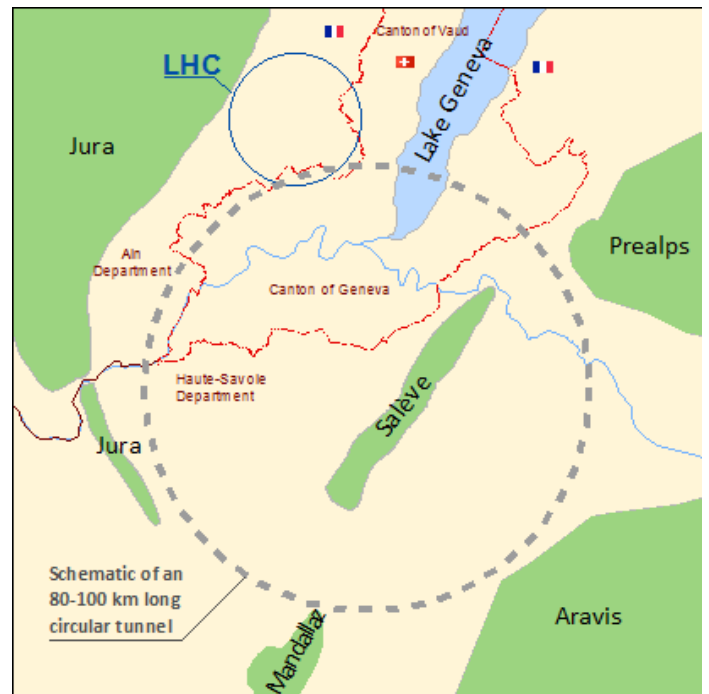

Figure 1. Schematic of a $100 \mathrm{~km}$ tunnel for a Future Circular Collider in the Lake Geneva basin [1].

The machine will accommodate two main proton experiments that are operated simultaneously but the

* Corresponding author: angelo.infantino@cern.ch 
layout allow for two additional special-purpose experiments. The machine will deliver a peak luminosity of $5-25 \cdot 10^{34} \mathrm{~cm}^{-2} \mathrm{~s}^{-1}$ [3]. The machine is compatible with ion beam operation. The main baseline features of FCChh are reported in Table 1. The realisation of a machine with $100 \mathrm{~km}$ circumference poses an enormous challenge in the availability and reliability of all kind of systems, since interventions and repairs would imply long downtime.

Table 1. FCC-hh baseline parameters compared to LHC and

\begin{tabular}{|c|c|c|c|}
\hline & $\mathrm{LHC}$ & $H L-L H C$ & $F C C-h h$ \\
\hline c.m. Energy [TeV] & & 14 & 100 \\
\hline Circumference $[\mathrm{km}]$ & & 26.7 & 100 \\
\hline Dipole Field [T] & & 8.33 & 16 \\
\hline Number of interaction points & & $2+2$ & $2+2$ \\
\hline Injection Energy [TeV] & & 0.45 & 3.3 \\
\hline Peak Luminosity $\left[10^{34} \mathrm{~cm}^{-2} \mathrm{~s}^{-1}\right]$ & 1 & 5 & 5 \\
\hline Optimum run time $[\mathrm{h}]$ & 15.2 & 10.2 & 12.1 \\
\hline Bunch Population $\left[10^{11}\right]$ (25ns) & 1.15 & 2.2 & 1.0 \\
\hline Number of bunches (25 $\mathrm{ns}$ ) & & 2808 & 10600 \\
\hline Beam Current [A] & 0.584 & 1.12 & 0.5 \\
\hline Stored Energy per beam [GJ] & 0.392 & 0.694 & 8.4 \\
\hline
\end{tabular}

\subsection{Radiation to electronics studies for FCC}

Radiation to Electronics (R2E) represents a crucial issue to be taken into account as design criteria of any high energy and intensity machine. Radiation effects in electronic devices can be divided into two main categories: cumulative effects and Single Event Effects (SEE). Cumulative effects, proportional to Total Ionizing Dose (TID), are due to damage induced by ionizing radiation and displacement atoms: the accumulation of defects causes measurable effects that can ultimately lead to device failure. On the other hand, SEE, proportional to High Energy Hadrons fluence (HEH, i.e. hadrons $>20$ $\mathrm{MeV}$ ), are due to the direct or indirect ionization by a single particle, able to deposit sufficient energy in order to disturb the operation of the device. They can only be characterized by their probability to occur, given their stochastic nature, which strongly depend on the device, the intensity and the kind of radiation filed [4]. The risk of failure of the before mentioned SEE becomes high as electronic components (e.g. logic, power devices) are present in a growing number of equipment. It is important to note that the radiation environment encountered in a high energy and intensity accelerator, the high number of electronic systems and components exposed to radiation, as well as the actual impact of radiation-induced failures on the machine operation, pose challenges not common in any other field.

In this context, in 2007 CERN decided to create a dedicated R2E task force (R2E-project) which consists of experts in various fields related to electronics damage: indeed, the study of the electronics sensitivity to radiation requires a multi-disciplinary approach, spanning from the knowledge of the electronic components, to the radiation environment, and to the physics models that describe the interaction of the radiation with matter.

In the past, a considerable amount of work has been done in R2E-oriented studies for LHC [4-7]. In this context, thanks also on the extensive experience maturated for LHC, the CERN's R2E project is currently involved in the analysis of the critical areas for electronics for FCC. As required for the LHC, FCC will need a significant amount of electronic components in the accelerator tunnel and in the side galleries to control and monitor the various infrastructures and systems: given that, an a priori evaluation of the radiation environment, the technology that would be required, the failure rate and the possible mitigation actions become strategic for a long-term planning.

For a high intensity and energy machine like FCC, typical sources of radiation are luminosity debris, direct losses on collimators and dumps, and beam interactions with the residual gas inside the vacuum chamber all along the accelerator; in addition, as regards FCC-ee, the main source of radiation in the tunnel is represented by the synchrotron radiation [8]. Moreover, the shape of the tunnel and the shielding thickness and configuration affects the radiation environment and consequently the effects on the electronic components. For these reason, the design of the FCC tunnel and the interaction regions becomes crucial for the choice of the technology to be used in the most important electronic components, e.g. control units or safety systems.

\subsection{The FLUKA code and the FCC model: scoring capabilities important for shielding and radiation damage to electronics studies}

In order to evaluate the impact of the radiation on the machine equipment, $\mathrm{MC}$ simulation represents an indispensable tool but it needs to rely both on a refined implementation of physics models of the particle interaction with matter and an accurate 3D-description of the region of interest [8].

In this context, FLUKA $[9,10]$ is a well benchmarked, multi-purpose and fully integrated particle physics Monte Carlo code for calculations of particle transport and interactions with matter, covering an extended range of applications like for example proton and electron accelerator shielding, target design, calorimetry, activation and dosimetry, cosmic ray studies, and radiotherapy.

FLUKA is constantly employed in the majority of CERN technical and engineering applications such as machine protection issues, energy deposition calculations and material damage to accelerator elements, or shielding design. In R2E context, FLUKA has been extensively employed in the past for studies of the radiation levels in critical areas for electronics in the LHC environment [4$8]$ as well as in modelling SEE $[11,12]$ demonstrating its capabilities in this field; recently it has been used in the FCC study $[13,14]$ for characterization of the radiation field in the FCC-hh detector.

\section{Aim of the work}

A conceptual design report for FCC will be delivered before the end of 2018, in time for the next update of the European Strategy for Particle Physics. In this context, 
this work will provide a first evaluation of the radiation environment in critical areas for electronics, based on FLUKA Monte Carlo (MC) simulation, for the FCC tunnel. In particular, a model of the tunnel in the arc section was created based on the original civil engineering (CE) studies already performed and further integrated in the existing FLUKA models of the beam line. Furthermore, the radiation environment were evaluated in critical areas for electronics such as electronic racks, power converter location and auxiliary tunnel.

Finally, first considerations on the impact of the radiation environment on electronics have been conducted for an a priori evaluation of possible relocation or mitigation actions.

\section{Materials and Methods}

FCC-hh aims to provide, compared to LHC, proton collisions a factor $\sim 7$ and $\sim 3$ higher in energy and bunches number respectively. The radiation levels are expected to scale with the beam intensity for direct losses, with luminosity for collision debris, and with both beam current and residual gas density for beam-gas interactions. At the same time the higher energy will lead to a further increase. While direct losses or collisions debris will be typically the dominating sources of radiation for specific $\mathrm{FCC}$ areas, a significant contribution from beam-gas interactions is expected in the large part of the accelerator due to the extension of the arc segments.

An analytical scaling of the radiation levels in a given section of the machine may result generally unreliable given the competition of multiple sources of radiations. In the arc section, a tentative analytical scaling can be performed given that beam-gas interaction represents the dominant source of radiation: a projection of the radiation levels in the FCC arc cell can be performed, in first approximation, by a scaling from LHC data taking into account the different energy, the beam current and the residual gas-density profile within the vacuum chamber. The energy can be scaled using the approach proposed by Stevenson [15] where the energy scales as a power of the ratio of the energy to a reference energy (i.e. LHC beam energy). The current, as reported in Table 1, is almost the same for the two machines. The residual gas-density profile of LHC, in the arc section, is unknown: generally, the gas-density in the LHC arc is considered to be $\leq 10^{9}$ $\mathrm{H}_{2}$ molecules per $\mathrm{cm}^{3}$. Due to the lack of knowledge of the residual gas-density profile in the LHC arc, we decided to go through a detailed MC simulation of the FCC machine. Indeed, in opposition to analytical methods, MC simulation allows for an accurate modelling of the particle transport but it needs to rely both on a refined implementation of physics models of the particle interaction with matter and an accurate 3Dmodelling of the region of interest [8].

In the present work, FLUKA simulation was used for a detailed modelling of the radiation environment in the FCC arc taking into account all the main parameters that can affect the radiation field such as the expected gas- density profile along the beam orbit inside the vacuum chamber; the beam optics; the accurate geometric and material reproduction of all the main elements of the generic arc cell including dipoles, quadrupoles, tunnel elements.

At CERN, a very detailed library of materials and geometry descriptions is available for accelerator elements such as dipoles, quadrupoles, collimators, etc. Based on this library, the baseline parameters and the most up-to-date studies on the beam optics, the generic arc cell was built, as shown in Figure 2. The generic arc cell is made of 12 bending dipoles and 2 quadrupoles (six dipoles followed by one quadrupole). Each dipole is 14.3 $\mathrm{m}$ long while the quadrupoles are $6.9 \mathrm{~m}$ long: the full arc cell has a total length of $213 \mathrm{~m}$. A detailed model of the tunnel was created based on the current layout designed by the CE. The cross section of the tunnel, $6 \mathrm{~m}$ in inner diameter, is shown in Figure 3.

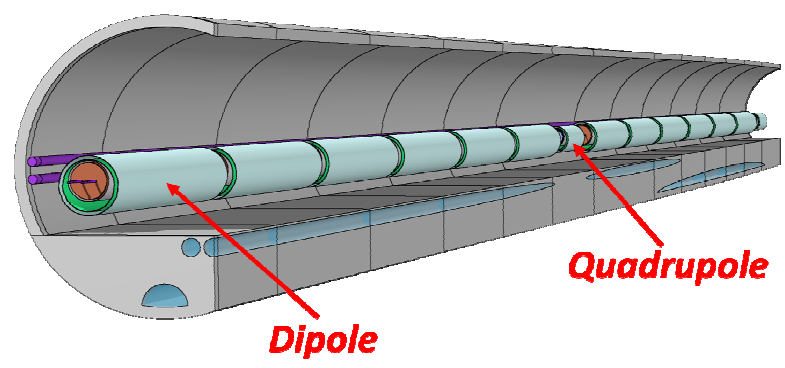

Figure 2. 3D-modelling of the FCC FLUKA arc cell: the cell is made of 12 bending dipoles and 2 quadrupoles, labelled in the following B1-B12 and Q1-Q2 respectively.

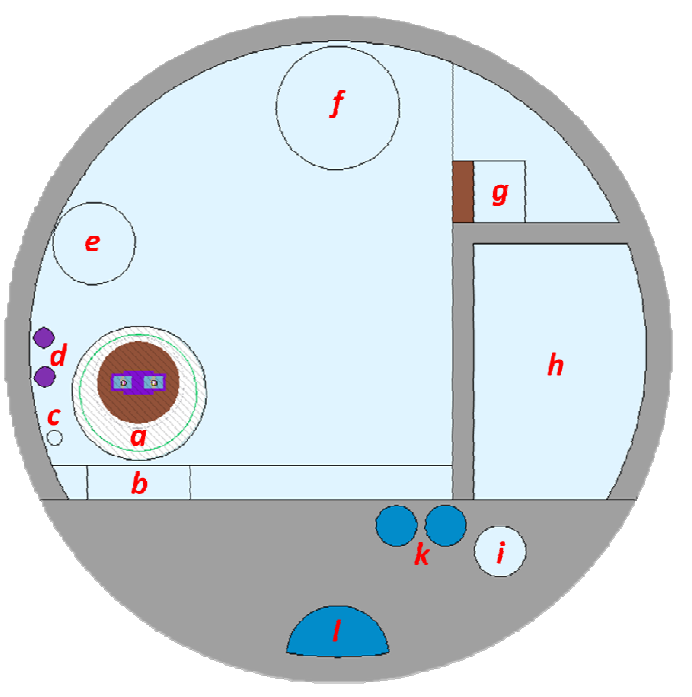

Figure 3. Cross section of the FLUKA model of current version of the FCC tunnel. The main elements included in the tunnel are a) dipole, b) power converter location, c) compressed air pipe, d) Helium recovery lines, e) cryo line, f) emergency extraction duct, g) electronics rack location, h) safe passage tunnel, i) safe passage ventilation, $\mathrm{k}$ ) cooling water pipes, 1) drainage.

Beam-gas interactions are considered the main source of secondary radiations in the arc section. A special source routine, already used in the past from our group [13], was used to allow for beam-gas interactions along the beam pipe. A tentative gas-density profile along the 
beam orbit inside the vacuum chamber, based on the most up-to-date studies from the CERN's vacuum group ( $R$. Kersevan, private communication), was implemented as shown in Figure 4: the gas-density increases at beam vacuum interconnects while it was considered substantially constant within the magnets.

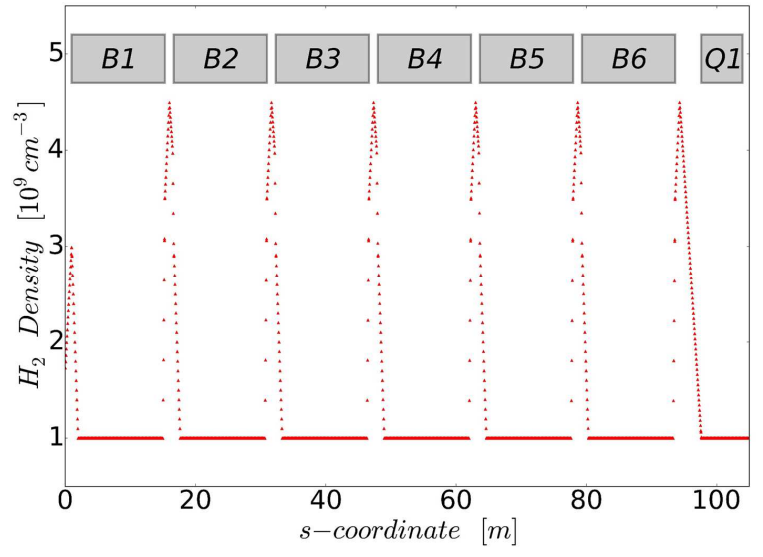

Figure 4. Tentative gas-density profile sampled in the FLUKA model. The gas-density profile is given in $\mathrm{H}_{2}$ molecules per unit volume as a function of the curvilinear s-coordinate. The picture shows only half-cell, including the first six bending magnets and the first quadrupole.

Both the clockwise and anticlockwise $50 \mathrm{TeV} / \mathrm{c}$ proton beam were simulated to consider real operation conditions. High energy hadron-nucleus interaction ( $>20$ $\mathrm{TeV} / \mathrm{c}$ ) are treated in FLUKA through the DMPJET [16] event generator; the full electromagnetic transport allow for an accurate scoring of the energy deposition in the areas critical for electronics. Due to the beam energy the calculation time needed was a bottleneck. Indeed, a hadronic nuclear interaction at FCC energies, like for LHC, can end in a very complex particle shower with hundreds of secondary: thus to simulate both the hadronic cascade and the electromagnetic shower in bulk matter may require a huge CPU effort. As the focus of this paper is on radiation effects on electronics, the simulations was optimized, in terms of physical and transport parameters, based on what is reported in [13]. To allow for a reasonable compromise between the CPU time per primary particle and the final statistical uncertainty, leading particle biasing on the electromagnetic shower $\left(\mathrm{e}^{-}\right.$ $/ \mathrm{e}^{+} / \gamma<1 \mathrm{GeV}$ ) and multiplicity biasing on the hadronic cascade were enabled. In addition to the optimization options mentioned above, the simulation was split into multiple jobs and distributed, on different CPUs, to the CERN's cluster: a total of 120.000 primary protons were simulated.

As mentioned in Section 1.2, both cumulative and stochastic effects lead to possible radiation damage to electronics. TID was scored in our model through a dedicated score in the location $b, g, h$ of Figure 3. The SEE rate is estimated by calculating the $\mathrm{HEH}$ fluence distribution in energy and convoluting it with a measured (or estimated) probability of observing SEE in the device of interest. FLUKA allows for a direct scoring of $\mathrm{HEH}$ fluence above $20 \mathrm{MeV}$ [4]. Different track-length density estimators were used to assess the differential particle fluence distributions in energy in the location $b, g, h$ of
Figure 3: estimators were set in order to score $\mathrm{HEH}$, protons, neutrons, pions, kaons and muons.

Finally, TID and HEH fluence were scored along the whole tunnel using a coarse Cartesian mesh $5 \mathrm{~cm} \times 5 \mathrm{~cm} \times$ $100 \mathrm{~cm}$.

\section{RESULTS}

Figure 5 and Figure 6 show the assessment of the $\mathrm{HEH}$ fluence and the dose respectively from the coarse mesh set on the entire tunnel. The Cartesian mesh allows for a qualitative analysis of the radiation levels which, depending from the location of interest, can be evaluated in the range $10^{10}-10^{11} \mathrm{HEH} \mathrm{cm} \mathrm{cm}^{-2} \mathrm{y}^{-1}$ and $10^{1}-10^{2} \mathrm{~Gy}^{-1}$ respectively.

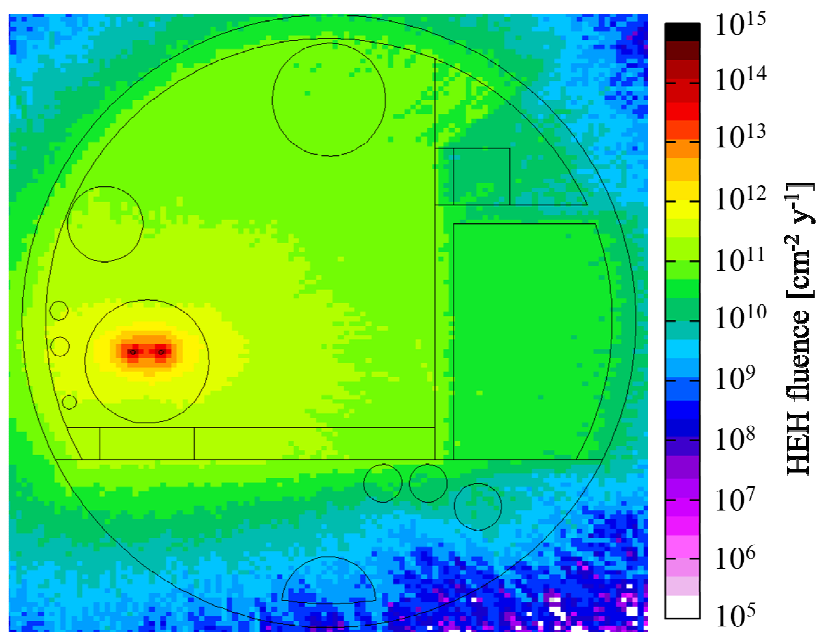

Figure 5. Assessment of the HEH fluence in the FCC tunnel. The slice is taken at the middle point of the bending magnet B1. A nominal FCC operational year of $10^{7} \mathrm{~s}$ was considered.

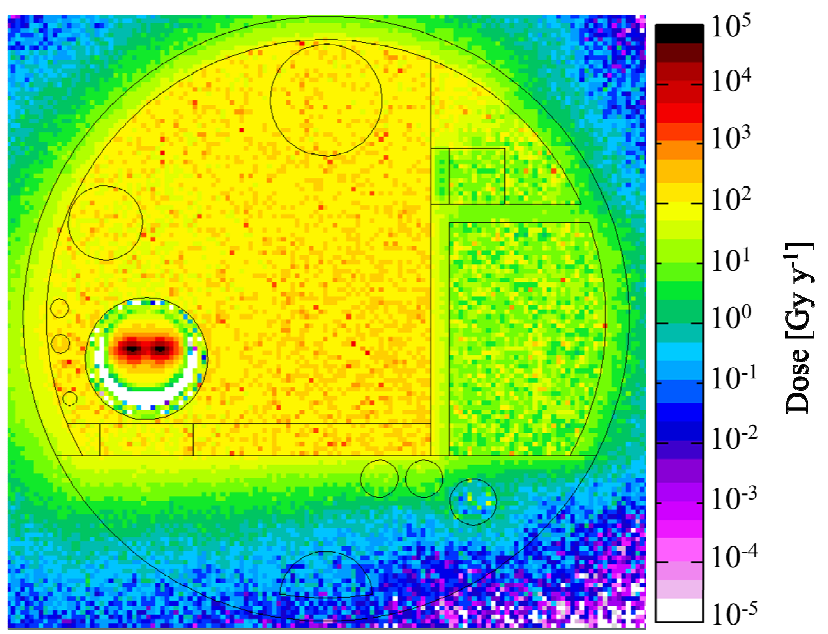

Figure 6. Assessment of the dose in the FCC tunnel. The slice is taken at the middle point of the bending magnet B1. A nominal FCC operational year of $10^{7} \mathrm{~s}$ was considered.

The dose and HEH fluence profile along the arc are shown in Figure 7 and Figure 8. Figure 7 shows how the dose increases at beam vacuum interconnects between two magnets where the gas-density increases and the macroscopic cross section of a beam-gas interaction is high. A similar trend can be found for the LHC [17], 
despite the fact that in [17] the gas-density profile was considered constant.

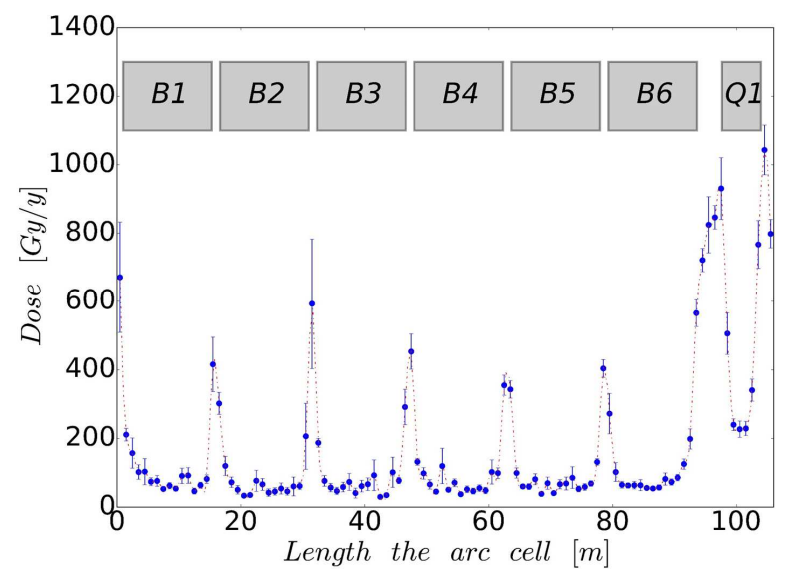

Figure 7. Dose profile (both beams) averaged along the arc in the power converter location (b). The picture shows only halfcell, including the first six bending magnets and the first quadrupole.

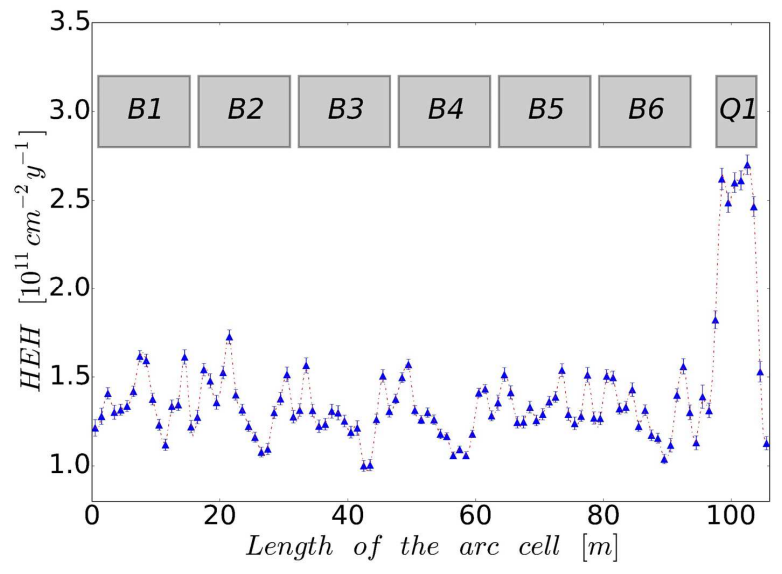

Figure 8. HEH fluence profile (both beams) averaged along the arc in the power converter location (b). The picture shows only half-cell, including the first six bending magnets and the first quadrupole.

Table 2. Radiation levels in the power converter location $(b)$, electronic rack location $(g)$, Safe Tunnel $(h)$.

\begin{tabular}{|c|c|}
\hline \multicolumn{2}{|c|}{ Power Converter (b) } \\
\hline Dose $\left[G y y^{-1}\right]$ & $(1.02 \pm 0.03) \mathrm{E}+02$ \\
\hline Protons $\left[\mathrm{cm}^{-2} \mathrm{y}^{-1}\right]$ & $(5.5 \pm 0.1) \mathrm{E}+09$ \\
\hline Kaons $\left[\mathrm{cm}^{-2} \mathrm{y}^{-1}\right]$ & $(6.5 \pm 0.3) \mathrm{E}+08$ \\
\hline Pions $\left[\mathrm{cm}^{-2} \mathrm{y}^{-1}\right]$ & $(6.2 \pm 0.1) \mathrm{E}+09$ \\
\hline Muons $\left[\mathrm{cm}^{-2} \mathrm{y}^{-1}\right]$ & $(1.17 \pm 0.05) \mathrm{E}+09$ \\
\hline Neutrons $\left[\mathrm{cm}^{-2} \mathrm{y}^{-1}\right]$ & $(1.25 \pm 0.01) \mathrm{E}+13$ \\
\hline $\mathrm{HEH}\left[\mathrm{cm}^{-2} \mathrm{y}^{-1}\right]$ & $(1.25 \pm 0.01) \mathrm{E}+11$ \\
\hline \multicolumn{2}{|c|}{ Electronic Rack (g) } \\
\hline Dose $\left[G y y^{-1}\right]$ & $(2.04 \pm 0.09) \mathrm{E}+01$ \\
\hline Protons $\left[\mathrm{cm}^{-2} \mathrm{y}^{-1}\right]$ & $(7.4 \pm 0.5) \mathrm{E}+08$ \\
\hline Kaons $\left[\mathrm{cm}^{-2} \mathrm{y}^{-1}\right]$ & $(7.1 \pm 0.1) \mathrm{E}+07$ \\
\hline Pions $\left[\mathrm{cm}^{-2} \mathrm{y}^{-1}\right]$ & $(5.1 \pm 0.3) \mathrm{E}+08$ \\
\hline Muons $\left[\mathrm{cm}^{-2} \mathrm{y}^{-1}\right]$ & $(3.5 \pm 0.4) E+08$ \\
\hline
\end{tabular}

\begin{aligned} & Neutrons $\left[\mathrm{cm}^{-2} \mathrm{y}^{-1}\right](3.74 \pm 0.04) \mathrm{E}+12 \\ &$ HEH $\left[\mathrm{cm}^{-2} \mathrm{y}^{-1}\right](2.00 \pm 0.06) \mathrm{E}+10 \\ &$\hline Safe Tunnel $(\boldsymbol{h}) \\ &$\hline Dose $\left.[\mathrm{Gy} \mathrm{y})^{-1}\right](1.63 \pm 0.04) \mathrm{E}+01 \\ &$ Protons $\left[\mathrm{cm}^{-2} \mathrm{y}^{-1}\right](1.60 \pm 0.06) \mathrm{E}+09 \\ &$ Kaons $\left[\mathrm{cm}^{-2} \mathrm{y}^{-1}\right](1.8 \pm 0.1) \mathrm{E}+08 \\ &$ Pions $\left[\mathrm{cm}^{-2} \mathrm{y}^{-1}\right](1.65 \pm 0.06) \mathrm{E}+09 \\ &$ Muons $\left[\mathrm{cm}^{-2} \mathrm{y}^{-1}\right](9.8 \pm 0.04) \mathrm{E}+08 \\ &$ Neutrons $\left[\mathrm{cm}^{-2} \mathrm{y}^{-1}\right](1.60 \pm 0.02) \mathrm{E}+12 \\ &$ HEH $\left[\mathrm{cm}^{-2} \mathrm{y}^{-1}\right](3.21 \pm 0.09) \mathrm{E}+10 \\ &$\hline\end{aligned}

The bi-dimensional dose and fluence map of Figure 5 and Figure 6 do not allow for a detailed analysis of the radiation levels: indeed, a quantitative analysis of the radiation levels can be performed through the dose and the particle fluence averaged over the regions of interest nearby a bending magnet (Table 2).

Finally, Figure 9, Figure 10 and Figure 11 show the detailed particle spectra in the region $b, g$, and $h$ respectively. The knowledge of the differential fluence distribution in energy allow, as mentioned above, to calculate the SEE rate: indeed, the SEE rate is estimated by calculating the HEH fluence distribution in energy and convoluting it with a measured (or estimated) probability of observing SEE in the device of interest [18]. Currently, we cannot calculate the SEE rate due to the lack of knowledge about the electronics that we are going to use in the future machine and its SEE cross section. Nevertheless, the analysis of the spectra can be useful to understand the particle environment at the different energies as well as the leading particles for SEE. With regard to protons, kaons, pions (and muons) the scoring was truncated at $20 \mathrm{MeV}$, as it possible to see from the unphysical cut in the spectra, given that for R2E purpose we are in interested in hadrons $>20 \mathrm{MeV}$ : indeed, below this threshold the Single Event Upset (SEU, i.e. the change of the state of a memory bit from 0 to 1 and vice versa) cross section falls down. The particle population is dominated by neutrons which can induce SEE in semiconductor devices through inelastic interactions in the sensitive volume. Furthermore, thermal neutrons, mainly generated through the slowing down of neutrons due to the interaction with the shielding materials, can contribute to SEE in electronics [19].

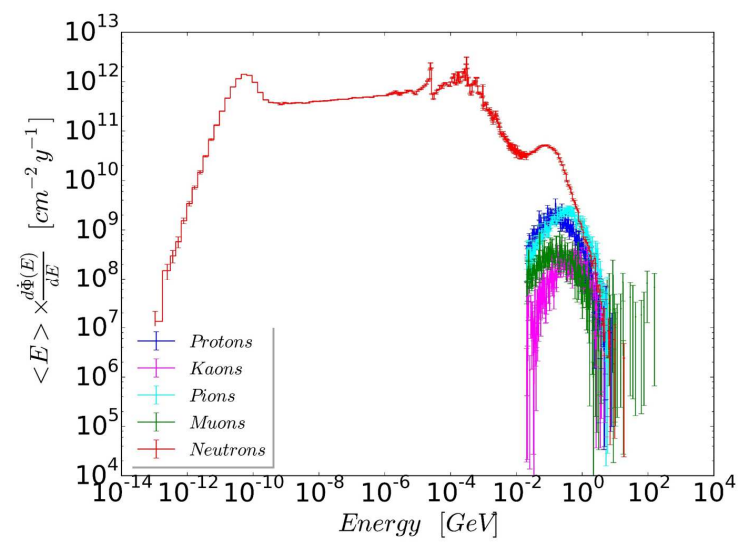

Figure 9. Particle spectra per unit lethargy in the power converter location $(b)$ 


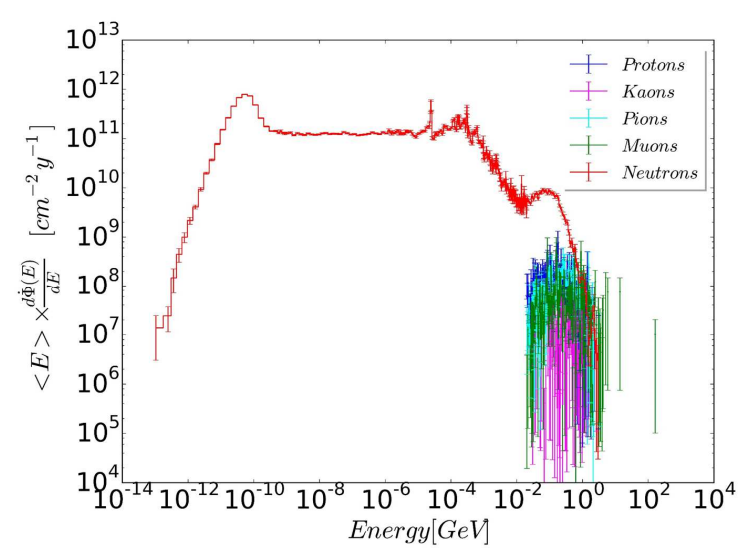

Figure 10. Particle spectra per unit lethargy in the electronic rack location $(g)$.

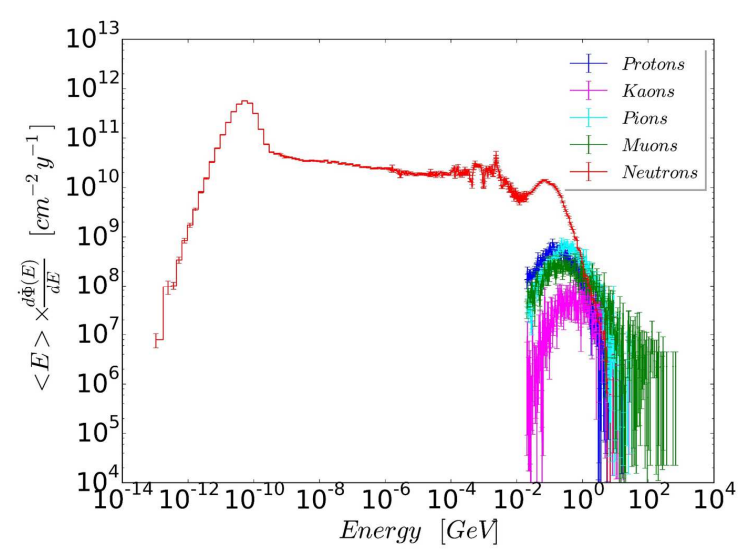

Figure 11. Particle spectra per unit lethargy in the safe tunnel passage $(h)$.

\section{CONCLUSION}

The FCC study is exploring the potential of hadronhadron and lepton-lepton circular colliders, for the postLHC era. The goal of FCC-hh is to provide proton-proton collisions at a centre-of-mass energy of $100 \mathrm{TeV}$. The need for building such large circular hadron collider is to reach energy levels far beyond the range of the LHC to provide access to the direct production of new particles with masses up to tens of $\mathrm{TeV}$, as well as to obtain much increased rates for phenomena in the sub-TeV mass range, with the corresponding greatly improved precision.

In this work, a first evaluation of the radiation levels in the FCC arc was discussed. In particular, the evaluation of the radiation levels was performed for critical areas for electronics.

An analytical scaling from LHC data could be performed in first approximation from the knowledge of the beam energy, the beam current and the residual gasdensity profile along the beam orbit in the vacuum chamber. The former, which is unknown for the LHC arc, cannot allow to perform a proper analytical scaling of the radiation levels. Moreover, such kind of scaling from the current machine may not be fully representative of the operational conditions of the future machine: indeed, unavoidable differences in the machine lattice, gasdensity profile, and physical mechanisms that might be accessible at higher energies are not taken into account and may lead to significant difference.

In opposition to analytical methods, MC simulation allows for an accurate particle transport, taking into account the actual geometry, the exact source term and all the main physical mechanisms. In this work, a FLUKA MC model of the FCC arc was developed considering the most up-to-date design of the tunnel layout, the beam optics, a tentative residual gas-density profile. MC simulations allowed to evaluate the TID and the HEH fluence in areas critical for electronics, namely the power converter location below the beam pipe, a dedicated electronic rack location and the safety tunnel. Considering the power converter area, the most critical of the three locations considered in this work, the expected TID is $(1.02 \pm 0.03) \mathrm{E}+02 \mathrm{~Gy}^{-1}$ while the $\mathrm{HEH}$ fluence is $(1.25 \pm 0.01) \mathrm{E}+11 \mathrm{~cm}^{-2} \mathrm{y}^{-1}$.

As a general conclusion, we do not know yet the technology that we are going to use in more than 20 years but considering that we expect an increment of the radiation levels of factors compared to LHC (i.e. increasing probability of SEE), the electronic components are going to be much more integrated (i.e. increasing sensibility to radiations), the number of electronics devices in FCC will be larger than in LHC (i.e. increasing probability of SEE) the need of dedicated shielded areas for the arc become strategic to increase the FCC reliability of factors.

Moreover, the results presented in this paper refer to the tentative residual gas-density profile used in the simulation: we can expect an improvement of the vacuum quality in time, as from the experience of LHC, which will reduce the TID and HEH.

Further considerations and a comparison with the LHC data for the arc imply more details about the actual residual gas-density profile along the beam orbit in the vacuum chamber. New simulations and a detailed data collection is ongoing.

\section{References}

1. M. Benedikt, F. Zimmermann, CERN-ACC-2016005, (2016)

2. R. Tomás, M. Benedikt, A.V. Bogomyagkov, L. Bottura, F. Cerutti, A. Ferrari, B. Haerer, B. Holzer, E. Jensen, M. Koratzinos, R. Martin, L. Medina, L.S. Esposito, D. Schulte, E. Todesco, J. Wenninger, S. White, F. Zimmermann, Nucl. Phys. B 00, 1-8 (2014)

3. A. Ball, M. Benedikt, L. Bottura, O. Dominguez, F. Gianotti, B. Goddard, P. Lebrun, M. Mangano, D. Schulte, E. Shaposhnikova, R. Tomas, F. Zimmermann, CERN FCC-1401101315-DSC, (2014)

4. G. Battistoni, V. Boccone, F. Broggi, M. Brugger, M. Campanella, M. Carboni, F. Cerutti, A. Empl, A. Fassò, A. Ferrari, A. Ferrari, E. Gadioli, M. V. Garzelli, D. Kramer, M. Lantz, E. Lebbos, A. Mairani, A. Margiotta, A. Mereghetti, C. Morone, S. 
Muraro, K. Parodi, V. Patera, M. Pelliccioni, L. Pinsky, J. Ranft, K. Roeed, S. Roesler, S. Rollet, P. R. Sala, M. Santana, L. Sarchiapone, M. Sioli, G. Smirnov, F. Sommerer, C. Theis, S. Trovati, R. Versaci, R. Villari, H. Vincke, H. Vincke, V. Vlachoudis, J. Vollaire, N. Zapp, Prog. Nucl. Sci. Tech. 2, 948-954 (2011)

5. B. Bellesia, M. Brugger, A. Ferrari, D. Kramer, R. Losito, S. Myers, M. Pojer, S. Roesler, M. Solfaroli, A. Vergara, S. Weisz, T. Wijnands, Proceedings of Chamonix 2009 workshop on LHC Performance, 160-170 (2009)

6. K. Røed, V. Boccone, M. Brugger, A. Ferrari, D. Kramer, E. Lebbos, R. Losito, A. Mereghetti, G. Spiezia, R. Versaci, IEEE Trans. Nucl. Sci. 58, 932938 (2011)

7. K. Røed, M. Brugger, G. Spiezia, CERN-ATS-Note2011-077 (2012)

8. L. S. Esposito, M. Brugger, F. Cerutti, A. Ferrari, R. Losito, Proceedings of HF2014, 157-159 (2014)

9. T.T. Böhlen, F. Cerutti, M.P.W. Chin, A. Fassò, A. Ferrari, P.G. Ortega, A. Mairani, P.R. Sala, G. Smirnov, V. Vlachoudis, Nucl. Dat. She. 120, 211214 (2014)

10. A. Ferrari, P.R. Sala, A. Fassò, J. Ranft, $C E R N$ 2005-10, INFN/TC_05/11, SLAC-R-773 (2005)

11. A. Infantino, R. García Alía, M. Brugger. IEEE Trans. Nuc. Sci. DOI: 10.1109/TNS.2016.2621238.

12. R. García Alía, B. Biskup, M. Brugger, M. Calviani, C.Poivey, K. Røed, F. Saigné, G. Spiezia, F. Wrobel. IEEE Trans. Nuc. Sci. 60 (4), 2469-2476 (2013).

13. M. I. Besana, F. Cerutti, L.S. Esposito, A. Ferrari, A. Lechner, A. Mereghetti, E. Skordis, Proceedings of the first Annual Meeting of the Future Circular Collider study (2015)

14. M. I. Besana, F. Cerutti, A. Ferrari, W. Riegler, V. Vlachoudis. Proceedings of IPAC2016, 1414-1417, (2016)

15. G. R. Stevenson, Rad. Prot. Dos. 96, 359-371 (2001)

16. S. Roesler, R. Engel, J. Ranft, Proc. Monte Carlo 2000 Conference, 1033-1038 (2001)

17. F. Cerutti. Radiation levels today and in the future. Available:

https://indico.cern.ch/event/308246/contributions/71 0485/attachments/588449/809908/R2EAvailWork.pd f [Accessed 25 October 2016]

18. JEDEC. JEDEC Standard No. 89A (JESD89A), (2006).

19. R. García Alía, S. Bonaldo, M. Brugger, D. Danzeca, F. Ferrari, A. Infantino, Y. Iwamoto, J. Mekki, C. Theis, A. Thorton. Proceedings of ICRS13 \& RPSD2016, Unpublished data, (2016) 